\title{
The role of carbon ion radiotherapy for unresectable locally recurrent rectal cancer: a single institutional experience
}

\author{
Xin Cai ${ }^{1,2 \dagger}$, Yueyao Du ${ }^{3 \dagger}$, Zheng Wang ${ }^{1,2}$, Ping $\mathrm{Li}^{1,2}$, Zhan Yu ${ }^{1,2}$, Qing Zhang ${ }^{1,2^{*}}$ and Zhen Zhang ${ }^{2,4^{*}}$
}

\begin{abstract}
Background: Treatment for locally recurrent rectal cancer after surgery is still a challenge. With the physical and biological advantages, carbon-ion radiotherapy (CIRT) could be a choice for these patients. The purpose of this study was to investigate the efficacy and safety of CIRT for unresectable locally recurrent rectal cancer in Chinese patients.

Methods: Date from 25 patients with unresectable locally recurrent rectal cancer treated by CIRT from July 2015 to April 2019 were analyzed retrospectively. The endpoints of this study were overall survival (OS), local control (LC) and acute and late toxicity.

Results: With the median follow-up of 19.6 (range 5.1-52.5) months, data of all 25 patients were collected. Median prescribed dose for tumor was 72Gy (relative biologic efficacy (RBE)) (range 48-75.6Gy (RBE)). The LC rates at 1 and 2 years were 90.4 and $71.8 \%$. Overall LC at 1- and 2-year were 76.2 and $30.5 \%$ for 9 patients whose prescribed tumor doses of CIRT< 66 Gy (RBE), 100 and 100\% for 16 patients whose prescribed doses of CIRT $\geq 66$ Gy (RBE). Patients received $\geq 66$ Gy (RBE) had obviously better $L C$ rates than those received $<66 \mathrm{~Gy}$ (RBE) ( $P=0.001$ ). The OS rates at 1 and 2 years were 82.9 and $65.1 \%$, respectively. No acute toxicity over grade 2 was observed, grade 3 late toxicity were observed in 3 patients: gastrointestinal toxicity $(n=1)$, neuropathy $(n=1)$, pelvic infection $(n=1)$. No Grade 4 or higher toxicity was observed.
\end{abstract}

Conclusion: Our study shows that CIRT is effective for unresectable locally recurrent rectal cancer patients with acceptable toxicity.

Keywords: Carbon-ion, Rectal cancer, Pelvic radiation, Local recurrence, Particle therapy

\section{Introduction}

Local recurrence (LR) presents a challenge after combined modality treatment for rectal cancer. Although multiple strategies including total mesorectal excision (TME), radiation therapy as well as chemotherapy has been shown to improve clinical outcomes, it remains a significant problem

\footnotetext{
* Correspondence: qing.zhang@sphic.org.cn; zhen.zhang@sphic.org.cn

${ }^{+}$Xin Cai and Yueyao Du contributed equally to this work.

'Department of Radiation Oncology, Shanghai Proton and Heavy lon Center, 4365 Kang Xin Road, Shanghai 201321, China

${ }^{2}$ Shanghai Engineering Research Center of Proton and Heavy lon Radiation Therapy, 4365 Kang Xin Road, Shanghai 201321, China

Full list of author information is available at the end of the article
}

in practice $[1,2]$, and about $4-15 \%$ of the patients with rectal cancer will suffer from LR [3-7].

Several studies have shown that LRs after definitive surgery occur in the central or posterior pelvis, with the presacral-perineal space being identified as the primary site of recurrence. Salvage surgical therapy remains the mainstay for these LR lesions, offering the best chance for cure. However, only $20-30 \%$ of patients receive a potentially curative operation due to its high rates of complication and operative mortality $[8,9]$. Radiotherapy may be one of the alternative treatment strategies that can be employed to the ones that surgical resection

(C) The Author(s). 2020 Open Access This article is licensed under a Creative Commons Attribution 4.0 International License, which permits use, sharing, adaptation, distribution and reproduction in any medium or format, as long as you give appropriate credit to the original author(s) and the source, provide a link to the Creative Commons licence, and indicate if changes were made. The images or other third party material in this article are included in the article's Creative Commons licence, unless indicated otherwise in a credit line to the material. If material is not included in the article's Creative Commons licence and your intended use is not permitted by statutory regulation or exceeds the permitted use, you will need to obtain permission directly from the copyright holder. To view a copy of this licence, visit http://creativecommons.org/licenses/by/4.0/ The Creative Commons Public Domain Dedication waiver (http://creativecommons.org/publicdomain/zero/1.0/) applies to the data made available in this article, unless otherwise stated in a credit line to the data. 
is unfeasible. As we know, a higher radiation dose to the area may increase local control. But LR disease treated by conventional external beam radiation (EBRT) is often limited by surrounding dose-limiting structures such as small bowel and bladder. Furthermore, LR disease may present with a large fraction of hypoxic cells, which are always resistant to conventional irradiation [10].

Compared with conventional photon therapies, particle radiotherapy, in particular carbon-ion radiotherapy (CIRT), has its unique physical and biologic advantages in the treatment of local recurrent rectal cancer [11]. From physical aspects, CIRT is characterized by improved dose distribution and minimization of dose to surrounding normal tissues. Numerous studies [12-14] has shown that CIRT is effective in treating various solid carcinomas. As a promising evolving modality in radiotherapy, CIRT has special biological advantage to overcome the radioresistance of hypoxic cells [15]. Previous clinical data further concluded that oxygen status was not the independent predictor of local control with the use of CIRT [16], which indicated that CIRT might be an effective way to increase radiosensitivity for local recurrent disease. The objective of this trial was to assess the effectiveness and safety of CIRT in the treatment of recurrent rectal cancer in Chinese patients.

\section{Materials and methods Patient eligibility}

From July 2015 until March 2019, 25 unresectable pelvic local recurrent rectal cancer patients were treated by CIRT. Initially, all the patients had pathologically confirmed rectal adenocarcinoma (including mucious adenocarcinoma and signet-ring cell carcinoma) and underwent a curative resection of their primary disease and regional lymph nodes, without gross or microscopic residual disease. Clinical recurrent diagnosis should be confirmed by biopsy or meet at least two of the three criteria: 1 ) relative tumor markers elevated; 2) hypermetabolic lesion in positron emission tomography (PET) imaging; 3) imaging follow-up revealed gradually enlargement of occupying lesion. Most of the patients (22 of 25 patients) had isolated pelvic or lymph node recurrence without distant metastasis verified by computed tomography (CT) or magnetic resonance imaging (MRI) or PET imaging. Among the 25 patients, 3 had received chemotherapy due to unresectable lesions of lung metastasis and evaluated as stable disease (SD) at least three months before the treatment of CIRT. Surgical resection of LR was evaluated as unfeasible by experienced surgeons. Patients were required to have Eastern Cooperative Oncology Group performance status $\leq 2$, life expectancy $\geq 12$ months, and pretreatment evaluation consisted of a complete history and physical examination, complete blood count $(\mathrm{CBC})$, renal and liver function tests, chest $\mathrm{CT}$, ultrasound of abdomen, and MRI/CT of the pelvis or whole body PET-CT.

The excluded criteria included: 1) a previous history of other malignancy; 2) receiving more than one prior radiotherapy in the same site or time to last radiotherapy is less than 1 year; 3 ) acute bacterial or fungal infection. If the lesion of the local recurrence was too close (less than $5 \mathrm{~mm}$ ) to vital organs (bladder, digestive tract) were also excluded.

This study was approved by the ethics committee. Before starting CIRT, patients were informed of their disease status, the risks and benefits and the expense of CIRT. Written informed consent was obtained from each subject.

\section{Carbon-ion radiotherapy}

All 25 patients received carbon-ion external irradiation by Siemens particle therapy device. Carbon-ion irradiation was performed daily, five days a week, with a total of 16 to 21 fractions in 22 to 30 days. The gross tumor volume (GTV) was defined as the area of contrast enhancement on T1-weighted MR-imaging. The clinical target volume (CTV) was defined as the GTV adding a safety margin of 5-10 mm adapted for organ at risk (OARs). The planning target volume (PTV) was defined as $5 \mathrm{~mm}$ margin added to the CTV.

The median GTV dose of CIRT was 72Gy (RBE)(4875.6Gy (RBE)) and prescribed to the 95\% isodose line. For 8 patients without prior radiotherapy, the prescribed doses for GTV were 57-72Gy (RBE), 19-20 fractions at 3, 3.3 or 3.6 Gy (RBE) per daily fraction. For other 17 patients with prior radiotherapy over 1 year before CIRT, dose given for CTV were 48-75.6Gy (RBE), 16-21 fractions at 3, 3.3 or $3.6 \mathrm{~Gy}$ (RBE) per daily fraction. For 18 patients (13 with prior radiotherapy) treated with SIB, the median dose for GTV in these 18 patients was $72 \mathrm{~Gy}$ (RBE) (range:57.6 to $75.6 \mathrm{~Gy}$ (RBE)), at 3.3 or 3.6Gy (RBE) per daily fraction in 16-21 fractions.

\section{Chemotherapy}

Concurrent chemotherapy was applied in 7 patients whose total GTV dose was less than 60Gy (RBE) (capecitabine: $825 \mathrm{mg} / \mathrm{m}^{2}$ twice daily, 5 days per week) and CIRT. Considering of the gastrointestinal toxicity caused by chemotherapy and CIRT, concurrent chemotherapy was not given to patients who received higher dose. Systemic treatment was recommended for patients as adjuvant treatment.

\section{Treatment results and evaluation of adverse events Follow-up}

All patients were followed up according to the protocol. The first follow-up is one month after CIRT and then patients were followed up every 3 months in the initial 2 
years and every 3-6 months thereafter. Post treatment evaluation included pelvic MRI or CT or PET scans, et al. Changes in the tumor diameter before and after treatment were evaluated in accordance with the Response Evaluation Criteria in Solid Tumors (RECIST) scoring system (version 1.0). Complete response (CR) was defined as disappearance of the target lesions. Partial response (PR) indicates at least a 30\% decrease in the sum of longest diameter (LD) of the target lesion, taking as reference the baseline sum LD. SD ranged from a $30 \%$ decrease to a $20 \%$ increase in size. Progressive disease (PD) was described as at least a $20 \%$ increase in the sum of the LD of the target lesion.

\section{Adverse effects}

Acute toxicity from treatment was classified according to the National Cancer Institute Common Toxicity (CTCAE) Version 4.03. Acute toxicity was defined as symptoms first occurring or lasting $<90$ days after the completion of radiotherapy.

\section{Statistics}

Time to locoregional failure and distant metastases was measured from the completion of EBRT until documented treatment failure. Local control (LC) was defined as the absence of notable local disease recurrence based on MRI/CT, and/or PET scans. Local recurrence indicated measurable lesions occurring in the irradiated tumor bed. The LC rate and survival rate curves were estimated using the Kaplan-Meier method. All statistical analyses were calculated by Stata 16.0 (StataCorp LP, College Station, TX, USA).

\section{Results}

\section{Clinical characteristics of patients}

From July 2015 to April 2019, 25 patients with 25 lesions were enrolled in this study. Detailed characteristics of the patients enrolled in this study are shown in Table 1. Median patient age was 53 years (range 32 to 72 ). Relapse locations included the presacral region $(n=11)$, pelvic sidewalls $(n=9)$, perineum $(n=4)$, and colorectal perianastomosis $(\mathrm{n}=1)$.

The median follow-up duration for all the patients was 19.6 months (range, 5.1-52.5 months). All patients completed the entire treatment course.

After CIRT, 3 patients with unresectable distant metastasis diagnosed before CIRT continued chemotherapy. Among the rest of 22 patients, 18 patients received at least 4 cycles of fluorouracil-based chemotherapy. 4 patients did not receive chemotherapy, among them 2 were considered not tolerant to chemotherapy. 1 patient with small tumor size was suggested to follow up only. 1 patient refused to receive chemotherapy.
Table 1 Patient characteristics

\begin{tabular}{|c|c|}
\hline Characteristics & No. of patients (\%) \\
\hline \multicolumn{2}{|l|}{ Age, years } \\
\hline Median & 53 \\
\hline Range & $32-72$ \\
\hline \multicolumn{2}{|l|}{ KPS } \\
\hline 80 & $7(28.0 \%)$ \\
\hline 90 & $14(56.0 \%)$ \\
\hline 100 & $4(16.0 \%)$ \\
\hline \multicolumn{2}{|l|}{ Gender } \\
\hline Male & $19(76.0 \%)$ \\
\hline Female & $6(24.0 \%)$ \\
\hline \multicolumn{2}{|l|}{ Primary tumor operation } \\
\hline abdominoperineal excision & $13(52.0 \%)$ \\
\hline low anterior resection & $10(40.0 \%)$ \\
\hline Hartmann's resection & $2(8.0 \%)$ \\
\hline \multicolumn{2}{|l|}{ Prior pelvic radiation therapy } \\
\hline Yes & $17(68.0 \%)$ \\
\hline No & $8(32.0 \%)$ \\
\hline \multicolumn{2}{|c|}{ Dose of prior pelvic radiation therapy } \\
\hline Median & $50 G y$ \\
\hline Range & 27-60Gy \\
\hline \multicolumn{2}{|l|}{ Tumor sites } \\
\hline presacral & $11(44.0 \%)$ \\
\hline side wall & $9(36.0 \%)$ \\
\hline perineal & $4(16.0 \%)$ \\
\hline perianastomosis & $1(4.0 \%)$ \\
\hline \multicolumn{2}{|l|}{ Tumor size } \\
\hline range & $6.1-334.1(\mathrm{ml})$ \\
\hline average & $84.5(\mathrm{ml})$ \\
\hline \multicolumn{2}{|l|}{ Total Dose of Carbon lon } \\
\hline <66Gy (RBE) & $9(36.0 \%)$ \\
\hline 2 66Gy (RBE) & $16(64.0 \%)$ \\
\hline
\end{tabular}

\section{Tumor response}

Treatment response was evaluated in all 25 patients (Table 2), four of 25 (16\%) had PR and twenty-one of 25 (84\%) patients had SD as soon as the treatment of CIRT was finished. Until the last follow-up in the end of March 2020, changes in the tumor were evaluated by image again and the results showed that $2(8 \%)$ and 7 (28\%) patients were evaluated as CR and PR, 10(40\%) patients were considered as SD, and 6(24\%) patients were considered as PD for their pelvic lesions. One of the patients defined as PR by image received surgery due to gastrointestinal toxicity, and the pathological report showed there was no tumor left and he achieved pathological complete response (pCR). Of the 16 patients with 
Table 2 Summary of clinical findings

\begin{tabular}{ll}
\hline Parameter & No. of patients (\%) \\
\hline Response $(\boldsymbol{n}=\mathbf{2 5})$ & $1(4.0 \%)$ \\
Pathologic complete response & $2(8.0 \%)$ \\
Clinical complete response & $6(24.0 \%)$ \\
Partial response & $10(40.0 \%)$ \\
Stable disease & $6(24.0 \%)$ \\
Progressive disease & \\
Symptom relief $(\boldsymbol{n}=\mathbf{1 6})$ & $9(56.3 \%)$ \\
Complete relief & $6(37.5 \%)$ \\
Partial relief & $1(6.2 \%)$ \\
No relief &
\end{tabular}

symptomatic response before CIRT, 15 (93.8\%) had improvement in their pain.

There were 3 patients who had distant metastases before the treatment of CIRT. During the follow-up, 9 of the other 22 patients developed distant metastases, among which 4 patients experience lung metastases, 3 patients experience bone metastases, 1 patient experience retroperitoneal lymph node metastasis and 1 patient experience both lung and bone metastases.

The 1- and 2-year LC rates were 90.4\% (95\% CI, 66.8 to $97.5 \%$ ) and $71.8 \%$ (95\% CI, 44.4 to $87.4 \%$ ) (Fig. 1). Overall LC at 1- and 2-year using the Kaplan Meier method were $76.2 \%$ (95\% CI, 33.2 to $93.5 \%$ ) and 30.5\% (95\% CI, 4.5 to $63.4 \%$ ) for patients whose prescribed tumor doses of CIRT< 66 Gy (RBE), 100 and $100 \%$ for patients whose prescribed doses of CIRT $\geq 66$ Gy (RBE). Patients received $\geq 66$ Gy (RBE) had obviously better LC rates than those received $<66 \mathrm{~Gy}(\mathrm{RBE})(P=0.001)$ (Fig. 2).
The 1- and 2-year LC rates were $86.2 \%$ (95\% CI, 55.0 to 96.4\%) for 17 patients who received re-irradiation for pelvic recurrence of rectal cancer.

The 1- and 2-year overall survival rate were $82.9 \%$ (95\% CI, 60.6 to $93.2 \%$ ) and $65.1 \%$ (95\% CI, 39.0 to 82.2\%) (Fig. 3). Overall survival rate at 1- and 2-year using the Kaplan Meier method were $77.8 \%$ (95\% CI, 36.5 to $93.9 \%$ ) and $55.6 \%$ (95\% CI, 20.4 to $80.5 \%$ ) for patients whose prescribed doses of CIRT $<66$ Gy (RBE), $86.5 \%$ (95\% CI, 55.8 to $96.5 \%$ ) and $69.2 \%$ (95\% CI, 25.5 to $90.6 \%$ ) for patients whose prescribed doses of CIRT $\geq 66$ Gy (RBE) (Table 3).

\section{Toxicity}

Acute and late toxicity of the 25 cases receiving CIRT are described in Table 4 . No grade $>3$ acute toxicities were observed. Grade 3 late toxicity were observed in 3 patients: gastrointestinal toxicity $(n=1)$, neuropathy $(n=1)$, pelvic infection $(n=1)$.

\section{Discussion}

The present study firstly reported the clinical outcomes of 25 patients treated with CIRT for pelvic recurrence of rectal cancer in Chinese patients. With a median followup of 19.6 months, the 2-year LC rate and OS rate were 71.8 and $65.1 \%$, respectively. CIRT achieved favorable LC and survival comparable to surgery. Otherwise, adverse events were self-limited during the treatment of CIRT, without any grade 4 or higher toxicity. The results are promising and suggest that CIRT is an optional therapy for patients with pelvic recurrence of rectal cancer.

Previous clinical results for locally recurrent rectal cancer (LRRC) treated by CIRT were reported by Heidelberg

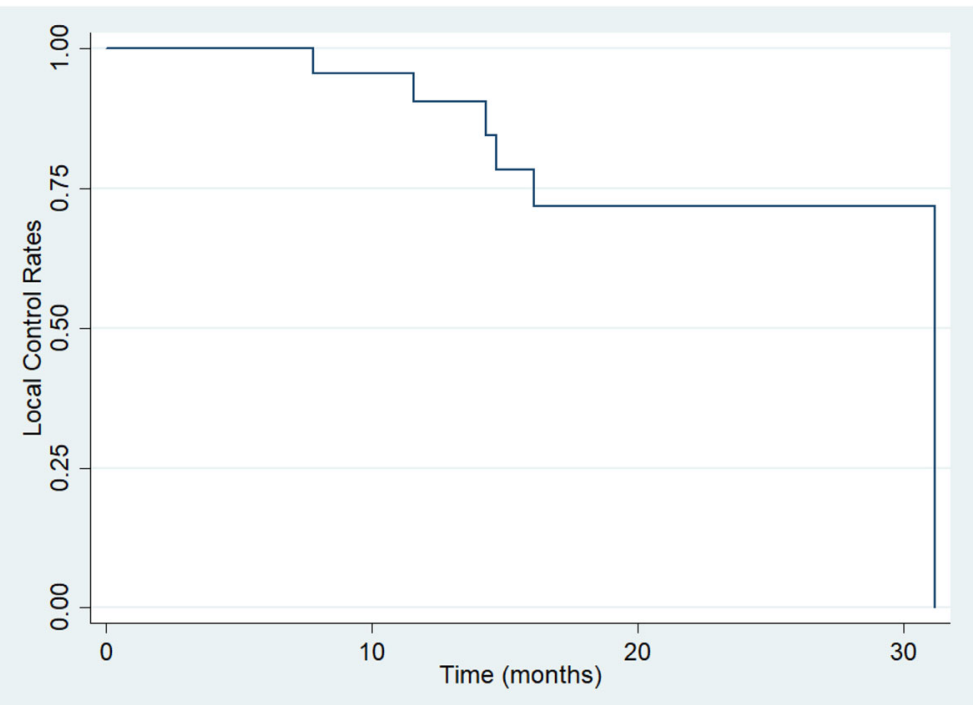

Fig. 1 Kaplan-Meier estimates of local control rate for the 25 patients with recurrent rectal cancer after the treatment of CIRT 


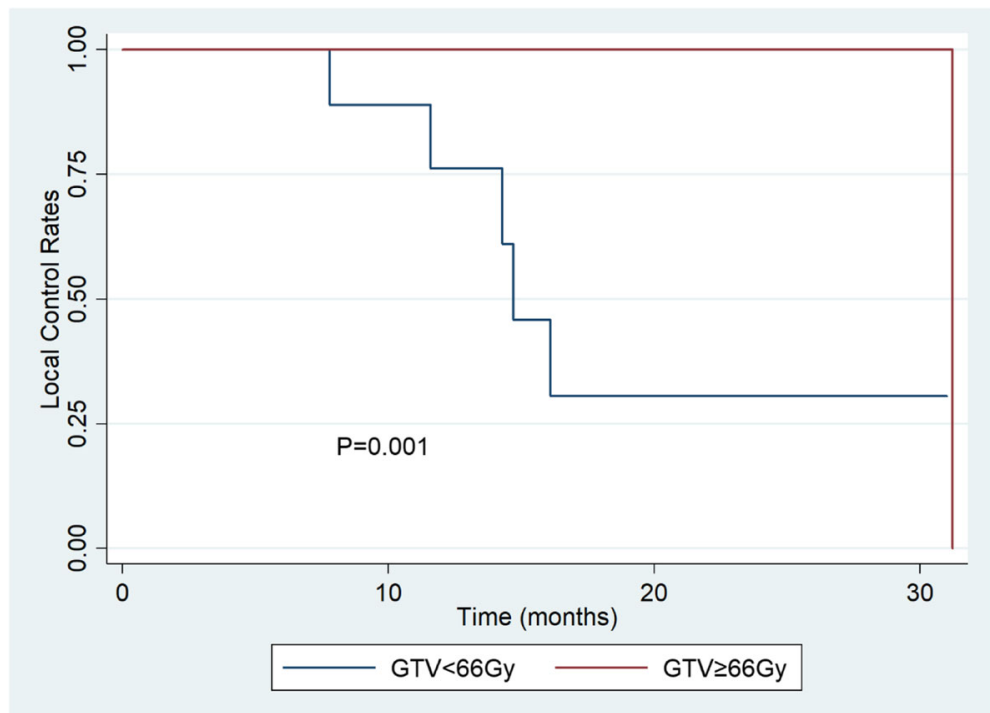

Fig. 2 Kaplan-Meier estimates of local control rate for patients whose prescribed tumor doses of CIRT<66 Gy (RBE) and $\geq 66$ Gy (RBE)

Ion Beam Therapy Center (HIT) in Germany and National Institutional of Radiological Sciences Hospital (NIRS) in Japan. In HIT's study, 19 patients received carbon ion irradiation to treat LRRC. All patients had a history of surgery and pelvic radiotherapy of at least 50.4Gy. The range of CIRT dose was 36-51Gy (RBE), 3Gy (RBE) per fraction. With median follow-up of 8 months, local progression-free survival was 20.6 months [14]. In NIRS's study [11], Shinoto et al. investigated the efficacy and safety of CIRT for LRRC. 224 patients were enrolled in the study and the prescribed dose was 70.4 or $73.6 \mathrm{~Gy}$ (RBE) in 16 fractions. The results showed that LC rates were $93 \%$ at 3 years and $88 \%$ at 5 years. OS rates were $73 \%$ at 3 years and $51 \%$ at 5 years. The prescribed dose of CIRT was relatively low and the follow-up duration was short in HIT's study. Compared with the results of the previous two studies, as the first study in patients receiving CIRT for LRRC in China, our study yielded acceptable and encouraging results in the efficacy of CIRT in treating LRRC.

For LRRC, there are other radiation modalities except CIRT. Cai et al. evaluated the efficacy and safety of irinotecan and capecitabine with concurrent intensitymodulated radiation therapy (IMRT) for the treatment of recurrent rectal cancer without prior pelvic irradiation.

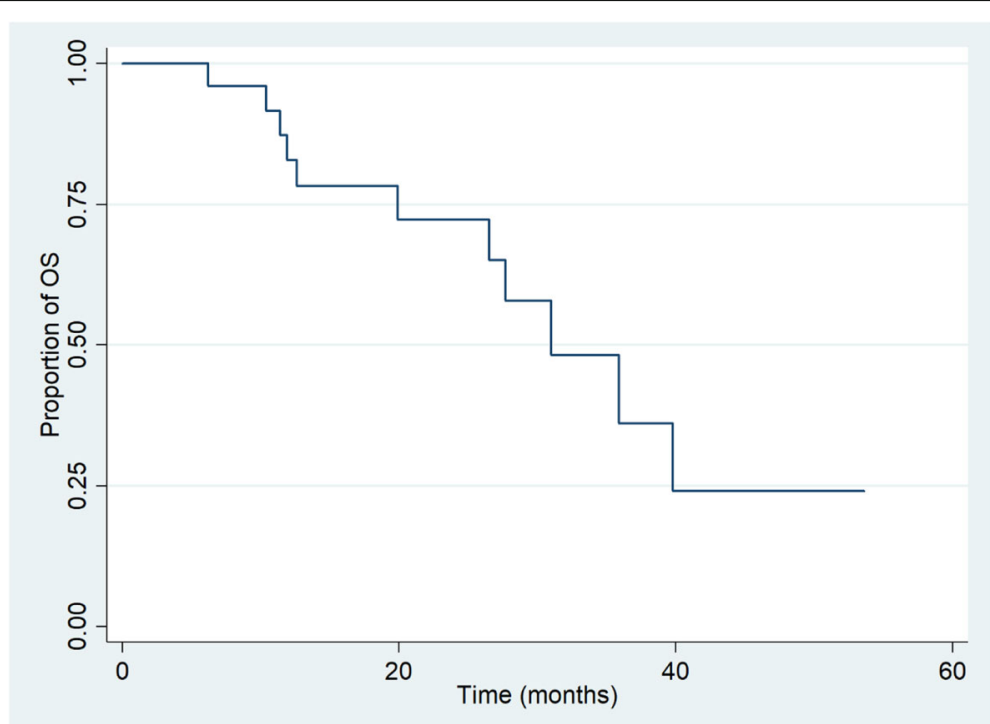

Fig. 3 Kaplan-Meier estimates of overall survival rate for the 25 patients with recurrent rectal cancer after the treatment of CIRT 
Table 3 Local control rate and overall survival rate of the patients receiving CIRT

\begin{tabular}{lll}
\hline & 1 year & 2 year \\
\hline LC & $90.4 \%$ & $71.8 \%$ \\
$<66$ (RBE) & $76.2 \%$ & $30.5 \%$ \\
$\geq 66$ (RBE) & $100 \%$ & $100 \%$ \\
OS & $82.9 \%$ & $65.1 \%$ \\
$<66$ (RBE) & $77.8 \%$ & $55.6 \%$ \\
$\geq 66$ (RBE) & 86.5 & $69.2 \%$ \\
\hline
\end{tabular}

Radiotherapy was delivered to the pelvis, and IMRT of 45 Gy (1.8 Gy per fraction), followed by a boost of $10 \mathrm{~Gy}$ to $16 \mathrm{~Gy}$, was delivered to the recurrent sites. For medically fit patients without extra-pelvic metastases, they would be recommended to receive radical surgery. After chemoradiation, the $\mathrm{LC}$ rates at 1 and 3 years were 74.2 and 33.9\%, the OS rates at 1 and 3 years were 80.1 and $36.5 \%$, respectively [17]. 2 patients experienced grade 4 leukopenia. No acute toxicity over grade 3 was reported. Sun et al. evaluated the efficacy and treatment-related toxicity of accelerated hyperfractionation field-involved re-irradiation combined with concurrent capecitabine chemotherapy for LRRC. Surgery would be performed after radiation if the disease was resectable. 3-year LC and OS were 31.19 and $45.12 \%$ [18]. Incidence of grade 3-4 diarrhea and granulocytopenia was 9.7 and $8.3 \%$. Small bowel obstruction was severely late toxicity, and the incidence was $1.4 \%$. Although the LC and OS rates in these two studies were acceptable, it should be noted that after IMRT and hyperfractionation re-irradiation, radical surgery would be performed if the lesion was evaluated as resectable. Defoe et al. evaluated the safety and efficacy of stereotactic body radiotherapy (SBRT) in LRRC patients with prior pelvic radiation. 11 patients were treated with 36 Gy in 3 fractions and 3 patients were treated with single fraction of 12,16 or 18 Gy. $1 y$ - and $2 y-\mathrm{LC}$ rates were 90.9 and $68.2 \%$ and the $1 \mathrm{y}$ - and $2 \mathrm{y}$ - OS rates were 90 and $78.8 \%$, respectively. No acute toxicity over grade 3 was observed [19]. Compared with conventional photon modalities, our results showed that CIRT was effective as a radical treatment modality for LRRC with or without prior pelvic radiation.
In our study, there was a significant correlation between dose and local control rates. Overall LC at 1- and 2-year using the Kaplan Meier method were 76.2 and $30.5 \%$ for patients whose prescribed tumor doses of CIRT< 66 Gy (RBE), 100 and 100\% for patients whose prescribed doses of CIRT $\geq 66$ Gy (RBE). Actually, among patients whose prescribed doses of CIRT $\geq 66$ Gy (RBE), only one patient progressed after 31.2 months' followup. In HIT's study [14], the results showed that applied dose (36Gy (RBE) vs. $\geq 36$ Gy (RBE), 20.2 vs. 15.2 months, respectively) were not predictive of local failure. In NIRS's study [11], no correlation between prescribed dose and local control was noted. It should be noted that in HIT's study, the applied does was relatively low and in NIRS's study, the prescribed dose were almost the same. Another concern was the accuracy of CIRT dose deliver to the primary area, while there were $\mathrm{RBE}$ variation along the full range of spread-out Bragg peak (SOBP) fields. We confirmed that our planning system already calculated the physics dose based on the different RBE weighting, which indicated that LC might not correlated with the LET-RBE ratio. It was reasonable that in our results, patients received $\geq 66$ Gy (RBE) had obviously better LC rates than those received < $66 \mathrm{~Gy}$ (RBE). It suggests that the currently applied dose of CIRT ( $\geq 66$ Gy) in our center is feasible for patients with LRRC.

The toxicities of CIRT in treating LRRC were mild in our study. Systemic treatment before and after CIRT was suggested for LRRC patients. With less hematological toxicities caused by CIRT, more systemic treatment could be applied to decrease distant metastases, which might have benefits for LRRC patients in OS. Tumors that were close to gastrointestinal tract might cause gastrointestinal toxicity and pelvic infection in our study. Neuroinjury was reported mostly in high biological equivalent dose (BED) radiation therapy such as SBRT and CIRT in Japan. One of the patients enrolled in our study suffered neuropathy 11 months after CIRT. Since our treatment planning system already concerned the RBE variation along the different SOBP location, and the physics dose was determined based on different RBE weighting, it was

Table 4 Acute and late toxicities of the patients receiving CIRT

\begin{tabular}{|c|c|c|c|c|c|c|c|c|c|c|c|c|}
\hline & $\begin{array}{l}\text { Acute } \\
\text { (NCl-CTC) }\end{array}$ & & & & & & Late (RTOG/EORTC) & & & & & \\
\hline Toxicity & $n$ & Gro & Gr1 & $\mathrm{Gr} 2$ & Gr3 & Gr4 & $n$ & Gro & Gr1 & Gr2 & Gr3 & Gr4 \\
\hline Skin & 25 & 22 & 1 & 2 & 0 & 0 & 25 & 22 & 3 & 0 & 0 & 0 \\
\hline Gl tract & 25 & 24 & 1 & 0 & 0 & 0 & 25 & 24 & 0 & 0 & 1 & 0 \\
\hline Neuropathy & 25 & 25 & 0 & 0 & 0 & 0 & 25 & 24 & 0 & 0 & 1 & 0 \\
\hline Pelvic infection & 25 & 25 & 0 & 0 & 0 & 0 & 25 & 24 & 0 & 0 & 1 & 0 \\
\hline Hematological & 25 & 9 & 11 & 5 & 0 & 0 & 25 & 25 & 0 & 0 & 0 & 0 \\
\hline Others & 25 & 24 & 1 & 0 & 0 & 0 & 25 & 25 & 0 & 0 & 0 & 0 \\
\hline
\end{tabular}


reasonable not to concern more dose variation to the target volume. By reviewing CT scan, we found that the injured nerve defined by electromyography was located at the high BED dose area. As high LET radiation, carbon ion would present with higher biological effects than Low LET irradiation, such as conventional radiotherapy. The fraction size for this patient was $\mathrm{xx}$, which would translate to a higher biological effective dose delivered to the target volume. Based on LQ model to predict the biologic effectiveness, the biologic equivalent dose of CIRT for this patient corresponded to approximately $\mathrm{xx}$ Gy of EBRT delivered in standard 2Gy fractionation. Therefore, we considered that the neuropathy might be due to the higher BED dose and we should pay more attention to the fractionated size of CIRT for organs at risk in the future plans.

As to the selection of patients with locally recurrent rectal cancer, surgery remains the major treatment for resectable diseases. All the participants enrolled in the present study were patients with unresectable locally recurrent rectal cancer and most of them received pelvic radiotherapy before. Considering the risk of re-irradiation, the lesion of the local recurrence should be at least $10 \mathrm{~mm}$ to vital organs (bladder, digestive tract). The results of the present study showed that patients received higher prescribed tumor doses of CIRT had obviously better LC rates. In the present study, the prescribed tumor dose of the first patient who achieved CR was 66 Gy (RBE), and it was the reason why we chose $66 \mathrm{~Gy}$ (RBE) as a cut-off point. Optimal cut-off point needs to be further explored in the future study.

There are some limitations in the present study, which need to be acknowledged. First, this is a retrospective study with a relatively short follow-up time. Longer follow-up on clinical outcomes is needed. Second, the sample size of this study is small. Larger and well-designed prospective studies are required to further evaluate the role of CIRT in treating LRRC. Third, systemic treatment before or after CIRT might affect OS of LRRC patients, which was not further analyzed in this study.

To sum up, the results of the present study suggest that CIRT is effective for LRRC and can provide LC and OS rates that are comparable to those of the prior studies of CIRT. The incidence of acute and late toxicities was also tolerable. CIRT should be considered as a safe, effective treatment option for LRRC and can provide an alternative to surgery.

\footnotetext{
Abbreviations

CIRT: carbon-ion radiotherapy; OS: overall survival; LC: local control; RBE: relative biologic efficacy; LR: local recurrence; TME: total mesorectal excision; EBRT: external beam radiaton; PET: positron emission tomography; $\mathrm{CT}$ : computed tomography; MRI: magnetic resonance imaging; SD: stable disease; CBC: complete blood count; GTV: gross tumor volume; CTV: clinical target volume; PTV: planning target volume; RECIST: Evaluation Criteria in Solid Tumors; CR: complete response; PR: partial response; LD: longest
}

diameter; PD: progressive disease; CTCAE: National Cancer Institute Common Toxicity; pCR: pathological complete response; LRRC: locally recurrent rectal cancer; HIT: Heidelberg Ion Beam Therapy Center; NIRS: National Institutional of Radiological Sciences Hospital; IMRT: intensity-modulated radiation therapy; SBRT: stereotactic body radiotherapy; BED: biological equralent dose

\section{Acknowledgments}

We acknowledge all the investigators, patients and their families.

\section{Authors' contributions}

Zhen Zhang is responsible for editorial correspondence and have contributed to all of the following: (1) the conception and design of the study, and acquisition, analysis and interpretation of data, (2) contributing reagents/materials/analysis tools, (3) revising the article, (4) final approval of the version to be submitted. Xin Cai, Yueyao Du, Qing Zhang, Zhen Zhang, Zheng Wang, Ping Li and Zhan Yu have contributed to as the following: (1) acquisition, analysis and interpretation of data, (2) drafting and revising the article.

\section{Funding}

The research is supported by the Shanghai Municipal Commission of Health and Family Planning [grant number 20164Y0268]. The funders had no role in study design, data collection and analysis, decision to publish, or preparation of the manuscript.

\section{Availability of data and materials}

The data and materials of this study are available from the corresponding author on reasonable request.

\section{Ethics approval and consent to participate}

The study was approved by Ethics Committee of Shanghai Proton and Heavy lon Center.

\section{Consent for publication}

Not applicable.

\section{Competing interests}

None declared.

\section{Author details}

'Department of Radiation Oncology, Shanghai Proton and Heavy lon Center, 4365 Kang Xin Road, Shanghai 201321, China. ${ }^{2}$ Shanghai Engineering Research Center of Proton and Heavy Ion Radiation Therapy, 4365 Kang Xin Road, Shanghai 201321, China. 'Department of Breast Surgery, Renji Hospital, School of Medicine, Shanghai Jiao Tong University, Shanghai 200127, China. ${ }^{4}$ Shanghai Proton and Heavy Ion Center, Fudan University Cancer Hospital, 4365 Kang Xin Road, Shanghai 201321, China.

Received: 25 June 2020 Accepted: 20 August 2020

Published online: 28 August 2020

\section{References}

1. van den Brink M, Stiggelbout AM, van den Hout WB, Kievit J, Klein Kranenbarg E, Marijnen CA, Nagtegaal ID, Rutten HJ, Wiggers T, van de Velde CJ. Clinical nature and prognosis of locally recurrent rectal cancer after total mesorectal excision with or without preoperative radiotherapy. J Clin Oncol. 2004;22(19):3958-64. https://doi.org/10.1200/JCO.2004.01.023.

2. Bolognese A, Cardi M, Muttillo IA, Barbarosos A, Bocchetti T, Valabrega S. Total mesorectal excision for surgical treatment of rectal cancer. J Surg Oncol. 2000;74(1):21-3.

3. Kapiteijn E, Marijnen CA, Colenbrander AC, Klein Kranenbarg E, Steup WH, van Krieken JH, van Houwelingen JC, Leer JW, van de Velde CJ. Local recurrence in patients with rectal cancer diagnosed between 1988 and 1992: a population-based study in the West Netherlands. Eur J Surg Oncol. 1998;24(6):528-35

4. Galandiuk S, Wieand HS, Moertel CG, Cha SS, Fitzgibbons RJ Jr, Pemberton $\mathrm{JH}$, Wolff BG. Patterns of recurrence after curative resection of carcinoma of the colon and rectum. Surg Gynecol Obstet. 1992;174(1):27-32.

5. Bozzetti F, Mariani L, Miceli R, Doci R, Montalto F, Andreola S, Gennari L. Cancer of the low and middle rectum: local and distant recurrences, and survival in 
350 radically resected patients. J Surg Oncol. 1996;62(3):207-13. https://doi.org/ 10.1002/(SICl)1096-9098(199607)62:3<207:AID-JSO11>3.0.CO;2-3.

6. Shinoto M, Terashima K, Suefuji H, Matsunobu A, Toyama S, Fukunishi K, Shioyama Y. A single institutional experience of combined carbon-ion radiotherapy and chemotherapy for unresectable locally advanced pancreatic cancer. Radiother Oncol. 2018;129(2):333-9. https://doi.org/10. 1016/j.radonc.2018.08.026.

7. Shiba S, Okamoto M, Kiyohara H, Ohno T, Kaminuma T, Asao T, Ojima H, Shirabe K, Kuwano H, Nakano T. Prospective observational study of high-dose carbon-ion radiotherapy for pelvic recurrence of rectal Cancer (GUNMA 0801). Front Oncol. 2019;9:702. https://doi.org/10.3389/ fonc.2019.00702.

8. Zhu XG, Li YH, Li XF, Cai Y. Intensity-modulated radiation therapy for pelvic oligo-recurrence from rectal cancer: long-term results from a single institution. Am J Transl Res. 2016:8(2):1265-72.

9. Jimenez RB, Hickey S, DePauw N, Yeap BY, Batin E, Gadd MA, Specht M, Isakoff SJ, Smith BL, Liao EC, Colwell AS, Ho A, Januzzi JL, Passeri J, Neilan T, Taghian AG, Lu HM, MacDonald SM. Phase II study of proton beam radiation therapy for patients with breast Cancer requiring regional nodal irradiation. J Clin Oncol:JCO1802366. 2019. https://doi. org/10.1200/JCO.18.02366.

10. Hockel M, Schlenger K, Hockel S, Aral B, Schaffer U, Vaupel P. Tumor hypoxia in pelvic recurrences of cervical cancer. Int J Cancer. 1998; 79(4):365-9.

11. Shinoto M, Yamada S, Okamoto M, Shioyama Y, Ohno T, Nakano T, Nemoto K, Isozaki Y, Kawashiro S, Tsuji H, Kamada T. Carbon-ion radiotherapy for locally recurrent rectal cancer: Japan carbon-ion radiation oncology study group (J-CROS) study 1404 rectum. Radiother Oncol. 2019;132:236-40. https://doi.org/10.1016/.j.radonc.2018.10.007.

12. Tsujii $\mathrm{H}$, Kamada T. A review of update clinical results of carbon ion radiotherapy. Jpn J Clin Oncol. 2012;42(8):670-85. https://doi.org/10.1093/ jjco/hys104.

13. Yamada S, Kamada T, Ebner DK, Shinoto M, Terashima K, Isozaki Y, Yasuda S, Makishima H, Tsuji H, Tsujii H, Isozaki T, Endo S, Takahashi K, Sekimoto M, Saito N, Matsubara H, Working Group on Locally Recurrent Rectal C. Carbon-ion radiation therapy for pelvic recurrence of rectal Cancer. Int J Radiat Oncol Biol Phys. 2016;96(1):93-101. https://doi.org/ 10.1016/j.jijobp.2016.04.022

14. Habermehl D, Wagner M, Ellerbrock M, Buchler MW, Jakel O, Debus J, Combs SE. Reirradiation using carbon ions in patients with locally recurrent rectal Cancer at HIT: first results. Ann Surg Oncol. 2015;22(6):2068-74. https://doi.org/10.1245/s10434-014-4219-z.

15. Wenzl T, Wilkens JJ. Modelling of the oxygen enhancement ratio for ion beam radiation therapy. Phys Med Biol. 2011;56(11):3251-68. https://doi.org/ 10.1088/0031-9155/56/11/006.

16. Nakano T, Suzuki Y, Ohno T, Kato S, Suzuki M, Morita S, Sato S, Oka K, Tsujii $\mathrm{H}$. Carbon beam therapy overcomes the radiation resistance of uterine cervical cancer originating from hypoxia. Clin Cancer Res. 2006;12(7 Pt 1): 2185-90. https://doi.org/10.1158/1078-0432.CCR-05-1907.

17. Cai G, Zhu J, Palmer JD, Xu Y, Hu W, Gu W, Cai S, Zhang Z. CAPIRIIMRT: a phase II study of concurrent capecitabine and irinotecan with intensitymodulated radiation therapy for the treatment of recurrent rectal cancer. Radiat Oncol. 2015;10:57. https://doi.org/10.1186/s13014-015-0360-5.

18. Sun DS, Zhang JD, Li L, Dai Y, Yu JM, Shao ZY. Accelerated hyperfractionation field-involved re-irradiation combined with concurrent capecitabine chemotherapy for locally recurrent and irresectable rectal cancer. Br J Radiol. 2012;85(101 1):259-64. https://doi.org/10.1259/bjr/ 28173562

19. Defoe SG, Bernard ME, Rwigema JC, Heron DE, Ozhasoglu C, Burton S. Stereotactic body radiotherapy for the treatment of presacral recurrences from rectal cancers. J Cancer Res Ther. 2011;7(4):408-11. https://doi.org/10. 4103/0973-1482.92000

\section{Publisher's Note}

Springer Nature remains neutral with regard to jurisdictional claims in published maps and institutional affiliations.

\section{Ready to submit your research? Choose BMC and benefit from:}

- fast, convenient online submission

- thorough peer review by experienced researchers in your field

- rapid publication on acceptance

- support for research data, including large and complex data types

- gold Open Access which fosters wider collaboration and increased citations

- maximum visibility for your research: over $100 \mathrm{M}$ website views per year

At BMC, research is always in progress.

Learn more biomedcentral.com/submissions 